\title{
Theoretical description of two- and three-particle interactions in single ionization of helium by ion impact
}

\author{
M. F. Ciappina and W. R. Cravero \\ CONICET and Departamento de Física, Universidad Nacional del Sur, Avenida Alem 1253, B8000CPB, Bahía Blanca, Argentina \\ M. Schulz \\ Physics Department and Laboratory for Atomic, Molecular and Optical Research, University of Missouri-Rolla, Rolla, \\ Missouri 65409, USA
}

\author{
R. Moshammer and J. Ullrich \\ Max-Planck-Institut für Kernphysik, Saupfercheckweg 1, 69117 Heidelberg, Germany
}

(Received 29 June 2006; published 3 October 2006)

\begin{abstract}
In this work we calculate doubly differential cross sections (DDCS) for single ionization of helium by highly charged ion impact. We study the importance of two-particle interactions in these processes by considering the cross sections as a function of all two-particle subsystems momenta. Experimental DDCSs were obtained recently from kinematically complete experiments on single ionization of $\mathrm{He}$ by $100 \mathrm{MeV} / \mathrm{amu} \mathrm{C}^{6+}$ and 3.6 MeV/amu $\mathrm{Au}^{24,53+}$ impact. Furthermore, we evaluated the importance of three-particle interactions by plotting the squared momenta of all three collision fragments simultaneously in a Dalitz plot. Using the first Born and distorted-wave approximations for fully differential cross sections, together with Monte Carlo integration techniques, we were able to reproduce the main features observed in experimental data and to assess the quality of the models implied by the different employed approximations.
\end{abstract}

DOI: 10.1103/PhysRevA.74.042702

PACS number(s): $34.50 . F a, 34.10 .+\mathrm{x}$

\section{INTRODUCTION}

Kinematically complete experiments on single ionization of atoms by fast heavy-ion impact have in the past decade become feasible using the cold-target recoil-ion-momentum spectroscopy (COLTRIMS) technique [2]. In these experiments momenta of all particles interacting in a single ionization process can be recorded. Since the introduction of this technique, fully differential cross sections (FDCS) have been studied, both experimentally and theoretically $[3,4]$, and even three-dimensional images of the FDCS have been reported [5].

Experimental FDCSs show the usual structures for ionization processes: a large binary peak close to the momentum transfer q direction, which correspond to "binary" collisions between the active electron and the projectile, and a smaller recoil peak in the vicinity of $\mathbf{- q}$ direction, originated by a double-scattering mechanism, i.e., the electron first collides with the projectile and afterward it is scattered off the target nucleus.

A large number of theoretical approaches have been developed to calculate single ionization FDCSs. They can be classified into two big groups: perturbatives and nonperturbatives. The simplest perturbative approach is the first Born approximation (FBA) in which the transition amplitude is expanded in powers of the interaction potential retaining only the first-order term [6]. The usually employed FBA can be considered as a two-body theory since the ionization process is described in terms of a single interaction between the incident projectile and the electron, i.e., the target nucleus remains passive. This approximation has shown successful results as long as the projectile charge-to-velocity ratio is smaller than 1 [7], although some discrepancies still remain unexplained [5]. Higher-order contributions of the Born series become more important when the velocity of the projectiles is slow or when highly charged ions are used in the experiment. However, the Born series has well-known convergence problems for long-range potentials, and the numerical implementation of even a second-order approach is quite difficult. Accordingly, the quality of the approximation is hard to assess and for this reason calculations beyond second order have not been performed [8-10].

An alternative perturbative approach is the continuumdistorted-wave (CDW) model proposed by Belkic [11] based on the work of electronic capture made by Cheshire [12]. Here the interaction potentials are essentially split into a long-range distortion and a short-range perturbation. Distorted wave functions (both initial and final) are built in such a way that they contain additional information about the particle interactions, being a solution of the Hamiltonian that includes the distortion potentials. In this way, Born seriesconvergence problems can be, at least formally, removed since the perturbation has a short range. The price to be paid is that these potentials become differential operators.

The CDW approach has the advantage of giving very good results by considering just the first order of the underlying perturbative series. After a promising beginning, some problems were found in the asymptotic conditions of the pure CDW ionization theory [13]. Crothers and McCann circumvented these problems with the development of the socalled continuum-distorted-wave-eikonal initial state (CDWEIS) model [14]. This theory has become one of the most successful theories in the field of atomic ionization for high and intermediate impact energy $[15,16]$ and further improvements of this theory have been reported (e.g., $[17,18]$ ). A particularly important refinement was the incorporation of all 
two-body pair interactions, including the interaction between the projectile and the target nucleus ( $N-N$ interaction) $[19,20]$. The early computational attempts employed semiclassical approaches for the inclusion of such $N-N$ interaction [21]. Nowadays other groups implement more sophisticated approaches, but with marginal results [22,23]. Nonperturbative approaches for ion-atom collisions include closecoupling theories and the classical trajectories Monte Carlo method (CTMC); this last one has been widely used for the calculation of ionization cross sections at intermediate impact energies $[24,25]$. We will not further consider these nonperturbative approximations in this work.

On the experimental side, a large amount of FDCSs data have become available and can be used to sensitively test the different theoretical models (see, e.g., Ref. [26] and references therein). Theoretical calculations for FDCSs for electrons ejected into the scattering plane (defined by the initial and final projectile momenta) have reproduced the main features in the experiments reasonably well in the case of light projectiles, but in the case of highly charged ion impact, relatively poor agreement was obtained.

One disadvantage of the FDCS is that it is very difficult to represent the data as a function of all three particle parameters simultaneously in a single spectrum. As a consequence it is not easy to understand from the usual representation of data the relative importance of two-particle interactions and to disentangle relative motion correlations among all three particles.

It has been recently proposed that Dalitz plots could be used as a representation tool to improve our qualitative understanding of three-particle collision dynamics [1]. Earlier, these graphs were used to study electronic correlations in triple ionization [27]. It is not particularly difficult to generate such plots using experimental data because they merely amount to a statistical rearrangement of the momentum distributions routinely measured in COLTRIMS experiments. However, theoretical Dalitz plots are considerably more difficult to produce due to the complicated transformations and integration procedures involved. Only recently, theoretical Dalitz plots have been presented for CTMC calculations for FDCSs [28].

In this work we report a theoretical study on the DDCS for single ionization of He by highly charged ion impact. The DDCS will be presented both in the form of Dalitz plots and in a more conventional way, namely, as a function of the momenta of the various particle pairs. In Sec. II we briefly describe the theories used to deal with the ionization process. The numerical calculations and its comparison with the experimental data available are presented in Sec. III. Finally, Sec. IV contains our conclusions.

\section{THEORY}

We treat helium single ionization as a single-electron process. We assume that in the final state the "active" electron moves in the combined Coulomb field of the impinging projectile and the residual target core. Electron residual-target ion interaction is modeled by a given effective charge, which takes into account the partial screening due to the passive electron, as is usually considered in the CDW-EIS approach [29]. Within this model, the long-range electron-projectile interaction is accounted for by a pure Coulomb distortion in the final state, and by asymptotic eikonal phase in the entrance channel [14]. The $N-N$ interaction is treated as a pure Coulomb interaction between a projectile with charge $Z_{P}$ and the target core charge, assumed to be $Z_{T}=1$. Further, the $N-N$ interaction is then included in the transition amplitude, through its multiplication by a semiclassical phase factor [21].

In the center-of-mass (c.m.) frame, the FDCS in energy and ejection angle of the electron, and the direction of the outgoing projectile are given by [30-32]

$$
\frac{d^{5} \sigma}{d E_{k} d \Omega_{k} d \Omega_{K}}=N_{e}(2 \pi)^{4} \mu^{2} k \frac{K_{f}}{K_{i}}\left|T_{i f}\right|^{2} \delta\left(E_{f}-E_{i}\right),
$$

where $N_{e}$ is the number of electrons in the atomic shell, $\mu$ is the reduced mass of the projectile-target subsystem, and $K_{i}\left(K_{f}\right)$ is the magnitude of the incident particle initial (final) momentum. The ejected electron's energy and momentum are given by $E_{k}$ and $k$, respectively. The solid angles $d \Omega_{K}$ and $d \Omega_{k}$ represent the direction of scattering of the projectile and the ejected electron, respectively. We use nonorthogonal Jacobi coordinates $\left(\mathbf{r}_{P}, \mathbf{r}_{T}\right)$ to describe the collision process. These coordinates represent the position of the active electron with respect to the projectile $\left(\mathbf{r}_{P}\right)$ and the target ion $\left(\mathbf{r}_{T}\right)$, respectively. $\mathbf{R}_{T}$ is also needed, representing the position of the incoming projectile with respect to the c.m. of the subsystem $e-T$. If we neglect terms of order $1 / M_{T}$ and $1 / M_{P}$, where $M_{T}$ is the mass of the target ion nucleus and $M_{P}$ is the mass of the incident heavy ion, we can write $\mathbf{R}_{T}=\mathbf{r}_{T}-\mathbf{r}_{P}$.

Within the prior form of CDW-EIS, the transition amplitude can be computed as

$$
T_{i f}^{C D W-E I S,(-)}=\left\langle\chi_{f}^{C D W,(-)}\left|W_{i}\right| \chi_{i}^{E I S,(+)}\right\rangle,
$$

where the initial- (final-) state distorted wave $\chi_{i}^{E I S,(+)}$ $\left(\chi_{f}^{C D W,(-)}\right)$ is an approximation to the initial (final) state, which satisfies the outgoing-wave (+) [incoming-wave (-)] conditions. For the initial state the asymptotic form of the Coulomb distortion (eikonal phase) is used in the electronprojectile interaction, together with a semianalytical Rothaan-Hartree-Fock (RHF) description for the initial bound-state wave function

$$
\chi_{i}^{E I S,(+)}=(2 \pi)^{-3 / 2} \exp \left(i \mathbf{K}_{i} \cdot \mathbf{R}_{T}\right) \psi_{R H F}\left(\mathbf{r}_{T}\right) \mathcal{E}_{v}^{+}\left(\mathbf{r}_{P}\right),
$$

where $\mathcal{E}_{v}^{+}\left(\mathbf{r}_{P}\right)$ is

$$
\mathcal{E}_{v}^{+}\left(\mathbf{r}_{P}\right)=\exp \left(-i \frac{Z_{P}}{v} \ln \left(v r_{P}-\mathbf{v} \cdot \mathbf{r}_{P}\right)\right)
$$

and

$$
\psi_{R H F}\left(\mathbf{r}_{T}\right)=\sum_{\lambda=1}^{5} \frac{Z_{\lambda}^{3 / 2} A_{\lambda}}{\pi^{1 / 2}} \exp \left(-Z_{\lambda} r_{T}\right) .
$$

The effective charges $Z_{\lambda}$ and the weights $A_{\lambda}$ can be obtained from Ref. [33].

On the other hand, the final-state wave function is cast into the form $[14,34,35]$ 


$$
\chi_{f}^{C D W,(-)}=(2 \pi)^{-3 / 2} \exp \left(i \mathbf{K}_{f} \cdot \mathbf{R}_{T}\right) \chi_{T}^{-}\left(\mathbf{r}_{T}\right) C_{P}^{-}\left(\mathbf{r}_{P}\right),
$$

where $C_{P}^{-}$represents the Coulomb distortion of the ejected electron wave function due to the projectile, i.e.,

$$
C_{P}^{-}\left(\mathbf{r}_{P}\right)=N\left(\nu_{P}\right)_{1} F_{1}\left(-i \nu_{P}, 1,-i k_{P} r_{P}-i \mathbf{k}_{P} \cdot \mathbf{r}_{P}\right),
$$

where $\nu_{P}=\frac{Z_{P}}{k_{P}}$ is the Sommerfeld parameter, $\mathbf{k}_{P}$ is the relative momentum of the $e-P$ subsystem, and $N\left(\nu_{P}\right)$ is the usual Coulomb factor

$$
N\left(\nu_{P}\right)=\Gamma\left(1-i \nu_{P}\right) \exp \left(\pi \nu_{P} / 2\right) .
$$

Furthermore, $\chi_{T}^{-}\left(\mathbf{r}_{T}\right)$ is the wave function for the ejected electron in the field of the residual target ion

$$
\begin{aligned}
\chi_{T}^{-}\left(\mathbf{r}_{T}\right)= & (2 \pi)^{-3 / 2} \exp \left(i \mathbf{k} \cdot \mathbf{r}_{T}\right) N\left(\nu_{T}\right)_{1} F_{1}\left(-i \nu_{T}, 1\right. \\
& \left.-i k r_{T}-i \mathbf{k} \cdot \mathbf{r}_{T}\right)
\end{aligned}
$$

with $\nu_{T}=\frac{Z_{T}}{k}$ and where $\mathbf{k}$ is the relative momentum of the $e$ $T$ subsystem.

The perturbation potential $W_{i}$ in Eq. (2) is defined by

$$
\left(H_{i}-E_{i}\right) \chi_{i}^{E I S,(+)}=W_{i} \chi_{i}^{E I S,(+)},
$$

where $H_{i}$ is the full electronic initial Hamiltonian (neglecting the total c.m. motion) and $E_{i}$ is the total initial energy of the system in the c.m. frame, respectively. $W_{i}$ is composed of two differential operators that can be written [36]

$$
W_{i}=\frac{1}{2} \nabla_{\mathbf{r}_{P}}^{2}-\nabla_{\mathbf{r}_{T}} \cdot \nabla_{\mathbf{r}_{P}}
$$

\section{A. Doubly-differential cross sections}

Equation (1) can be written alternatively,

$$
\frac{d^{5} \sigma}{d k d \Omega_{k} d \Omega_{K}}=N_{e}(2 \pi)^{4} \mu^{2} k^{2} \frac{K_{f}}{K_{i}}\left|T_{i f}\right|^{2} \delta\left(E_{f}-E_{i}\right)
$$

using that $d E_{k}=k d k$. Integrating Eq. (12) successively over ionized electron's angles $d \Omega_{k}=\sin \theta_{k} d \theta_{k} d \phi_{k}$ and projectile angle $\phi_{K}$ results

$$
\begin{aligned}
\frac{d^{2} \sigma}{d k \sin \theta_{K} d \theta_{K}}= & \int N_{e}(2 \pi)^{4} \mu^{2} k^{2} \frac{K_{f}}{K_{i}}\left|T_{i f}\right|^{2} \\
& \times \delta\left(E_{f}-E_{i}\right) \sin \theta_{k} d \theta_{k} d \phi_{k} d \phi_{K} .
\end{aligned}
$$

For small projectile angles $\sin \theta_{K} \approx \theta_{K}$ and consequently, $q_{\perp} \approx M_{P} v \theta_{K}=K_{i} \theta_{K}$. Rewriting Eq. (13), finally we obtain

$$
\begin{aligned}
\frac{d^{2} \sigma}{d k d q_{\perp}}= & \int N_{e}(2 \pi)^{4} \mu^{2} k^{2} q_{\perp} \frac{K_{f}}{K_{i}^{3}}\left|T_{i f}\right|^{2} \\
& \times \delta\left(E_{f}-E_{i}\right) \sin \theta_{k} d \theta_{k} d \phi_{k} d \phi_{K} .
\end{aligned}
$$

From energy conservation we know that $q_{\min }=q_{z}=\Delta \epsilon / v$, being $\Delta \epsilon=k^{2} / 2+\left|\epsilon_{i}\right|$ with $\epsilon_{i}$ the electron binding energy. Consequently, DDCS from Eq. (14) can be considered a function of $k$ and $q$, since $q=\sqrt{q_{\perp}^{2}+q_{z}^{2}}$. The usual method to calculate $\operatorname{DDCS}(q, k)$, as defined by Eq. (14), is to use quadratures in spherical polar coordinates. However, there are alternative approaches, such as Monte Carlo methods, to deal with integrations of the type of Eq. (14). These methods can be particularly convenient if we are interested in differential cross sections in terms of recoil-ion parameters, i.e., $\operatorname{DDCS}\left(p_{r}, k\right)$ and $\operatorname{DDCS}\left(q, p_{r}\right)$ where $p_{r}$ is the recoil-ion momentum.

\section{B. Dalitz plots}

When we analyze the experimental data available for DDCSs [1] we observe that the larger correlation emerges for binary interactions. This could be taken as strong support for the formulation of a final state as a product of two-body subsystems, as it is made in CDW and distorted-wave Born theories.

However, it is interesting to analyze to what extent the motion of all three collision fragments are correlated with each other. To do this, we will investigate the role of ionization events in which all three collision fragments undergo a significant change in momentum. Schulz et al. [1] have plotted their experimental data using Dalitz plots. In this way, the momenta of all three particles are embedded simultaneously in a single picture. When the dominant two-particle correlation is subtracted, their plots show significant contribution from events where strong interaction among the three particles takes place [1].

In the domain of particle physics, Dalitz plots have proved to be a very qualified tool to understand the decay modes of some three-body experiments [37]. Here, our aim is to obtain qualitative information regarding how well correlation is taken into account in theories used to calculate FDCSs. In a Dalitz plot for three particles of equal mass, the relative energy $\left(\varepsilon_{i}\right.$, with $\left.i=1,2,3\right)$ of each particle (normalized to the energy sum for all three particles) is plotted in an equilateral triangle. For a given experimental or theoretical point, the energy $\varepsilon_{i}$ for each particle is given by the perpendicular distance of that data point to the corresponding triangle side.

For ionization by ion impact we have three particles with different mass; consequently, this approach has to be slightly modified. Here, since the energy of the ion projectile is several orders of magnitude larger than for both the recoil ion and the ionized electron, the data would just concentrate in one point at the corner of the triangle opposite to the projectile side. To avoid this problem we follow Ref. [1] and plot the relative squared momenta instead of the relative energies. Consequently, our variables will be

$$
\begin{aligned}
& \pi_{e}=\frac{k^{2}}{k^{2}+q^{2}+p_{r}^{2}}, \\
& \pi_{q}=\frac{q^{2}}{k^{2}+p_{r}^{2}+q^{2}}, \\
& \pi_{r}=\frac{p_{r}^{2}}{k^{2}+q^{2}+p_{r}^{2}},
\end{aligned}
$$

where we have chosen the momentum transfer $\mathbf{q}$ for the projectile Dalitz plot's variable. It will be useful to plot the results to define a set of Cartesian coordinates $x_{D}$ and $y_{D}$ (see Fig. 1 for details), 


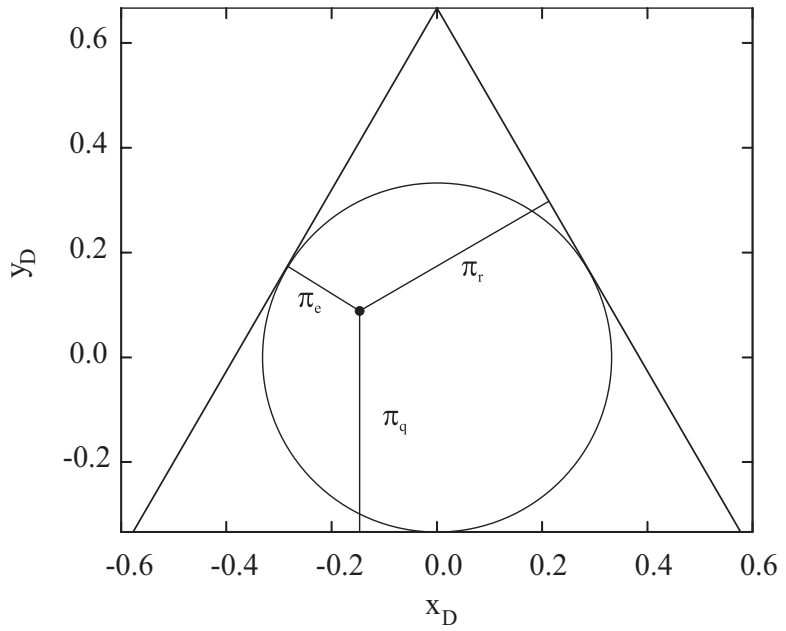

FIG. 1. Definition of the Dalitz plot for the ion-impact ionization case. See the text for details.

$$
\begin{aligned}
& x_{D}=\frac{\pi_{e}-\pi_{r}}{\sqrt{3}}, \\
& y_{D}=\pi_{q}-\frac{1}{3} .
\end{aligned}
$$

To some extent Dalitz plots define a type of DDCS, which is expressed as a function of $\pi_{e}, \pi_{q}$, and $\pi_{r}$ (note that $\pi_{e}+\pi_{q}$ $+\pi_{r}=1$, thus only two of the three variables are independent). The experimental and theoretical data must fall within the internal circle of the triangle because the areas outside that circle do not satisfy the kinematic condition $\mathbf{k}+\mathbf{p}_{r}=\mathbf{q}$ [1].

Starting from Eq. (15) and using momentum conservation, i.e., $\mathbf{q}=\mathbf{k}+\mathbf{p}_{r}$, we can write $\pi_{e}$ and $\pi_{q}$ as a function of $\mathbf{k}$ and $\mathbf{q}$,

$$
\begin{aligned}
& \pi_{e}=\frac{k^{2}}{2\left(k^{2}+q^{2}-\mathbf{k} \cdot \mathbf{q}\right)}, \\
& \pi_{q}=\frac{q^{2}}{2\left(k^{2}+q^{2}-\mathbf{k} \cdot \mathbf{q}\right)} .
\end{aligned}
$$

Accordingly, FDCS, i.e., Eq. (1), can be written in terms of the variables $\pi_{e}$ and $\pi_{q}$ as

$$
\frac{d^{5} \sigma}{d^{3} \mathbf{k} d q_{x} d q_{y}}=|J| \frac{d^{5} \sigma}{d^{3} \mathbf{k} d \pi_{e} d \pi_{q}},
$$

where $|J|$ represents the Jacobian of the transformation and we have written $d^{2} q_{\perp}=d q_{x} d q_{y}$. Explicitly, we obtain for $|J|$,

$$
|J|=\left|\frac{4 \pi_{e}^{3}}{k^{4}}\left(q_{x} k_{y}-q_{y} k_{x}\right)\right|
$$

where $k_{x}=k \sin \theta_{k} \cos \phi_{k}$ and $k_{y}=k \sin \theta_{k} \sin \phi_{k}$.

Using Eq. (21), the FDCS from Eq. (20) can be written

$$
\frac{d^{5} \sigma}{d^{3} \mathbf{k} d \pi_{e} d \pi_{q}}=\left|\frac{k^{4}}{4 \pi_{e}^{3}\left(q_{x} k_{y}-q_{y} k_{x}\right)}\right| \frac{d^{5} \sigma}{d^{3} \mathbf{k} d^{2} \mathbf{q}_{\perp}} .
$$

Now we need the transformation between $\left(q_{x}, q_{y}\right)$ [or, in polar coordinates, $\left.\left(q_{\perp}, \phi_{q}\right)\right]$ and $\left(\pi_{e}, \pi_{q}\right)$. These transformations have been included in the Appendix .

Finally, we obtain for the DDCS in terms of $\left(\pi_{e}, \pi_{q}\right)$,

$$
\begin{aligned}
\frac{d^{2} \sigma}{d \pi_{e} d \pi_{q}}= & \int\left|\frac{k^{4}}{4 \pi_{e}^{3}\left(q_{\perp} \cos \phi_{q} k_{y}-q_{\perp} \sin \phi_{q} k_{x}\right)}\right| \\
& \times \frac{d^{5} \sigma}{d^{3} \mathbf{k} d^{2} \mathbf{q}_{\perp}} d^{3} \mathbf{k},
\end{aligned}
$$

using that $q_{x}=q_{\perp} \cos \phi_{q}$ and $q_{y}=q_{\perp} \sin \phi_{q}$. As can be seen in the Appendix A, it is quite complicated to find the transformations $q_{\perp}\left(\pi_{e}, \pi_{q}\right)$ and $\phi_{q}\left(\pi_{e}, \pi_{q}\right)$ due to their nonlinear nature. In order to avoid the explicit use of these transformations, we employed Monte Carlo integration techniques taking the advantage of the simple relationship between $\mathbf{k}, \mathbf{q}$, and $\mathbf{p}_{r}$ in Cartesian coordinates.

\section{RESULTS AND DISCUSSION}

We can obtain information regarding the role of the twoparticle correlations within each collision subsystem analyzing the DDCSs as a function of the different particles momenta. We will then discuss the importance of three-particle interactions with the aid of theoretical Dalitz plots for ionimpact ionization of helium.

Following Ref. [1], in Fig. 2(a) we reproduce the experimental DDCS as a function of the projectile-momentum transfer $(q)$ and the magnitude of the electron momentum $(k)$, i.e., $\operatorname{DDCS}(q, k)$, for $100 \mathrm{MeV} / \mathrm{amu} \mathrm{C}^{6+}+\mathrm{He}$. In Figs. 2(b) and 2(c) the corresponding DDCSs are shown as a function of the recoil ion and electron momenta $\operatorname{DDCS}\left(p_{r}, k\right)$ and as a function of the projectile-momentum transfer and recoilion momentum $\operatorname{DDCS}\left(q, p_{r}\right)$, respectively. We have used a logarithmic scale based in the "counts" that appear in the experimental results (see Ref. [1] for details). Note that if the momentum of one particle is null, the momenta of the other two particles must have equal magnitude and opposite direction following momentum conservation. Therefore, ionization events, which are dominated by momentum exchange between any two particles would accumulate near the diagonal in the corresponding DDCS, forming a kind of "ridge."

Our first theoretical results for $100 \mathrm{MeV} / \mathrm{amu} \mathrm{C}^{6+}+\mathrm{He}$ are depicted in Fig. 3. Here we calculate and plot, using FBA approximation, $\operatorname{DDCS}(q, k)[$ panel (a) $], \operatorname{DDCS}\left(p_{r}, k\right)(\mathrm{b})$, and $\operatorname{DDCS}\left(q, p_{r}\right)(\mathrm{c})$. It can be seen from Fig. 2(b) that the strongest two-particle momentum exchange seems to occur within the recoil-ion-electron subsystem and our theoretical approach reproduces this feature reasonably well [Fig. 3(b)]. As we are interested in showing qualitatively the properties of our theories, we also use a logarithmic scale, but its magnitude can differ substantially from the experimental data scale. Take into account that the experimental plots were obtained directly from the "crude" data and consequently, they are not normalized. 

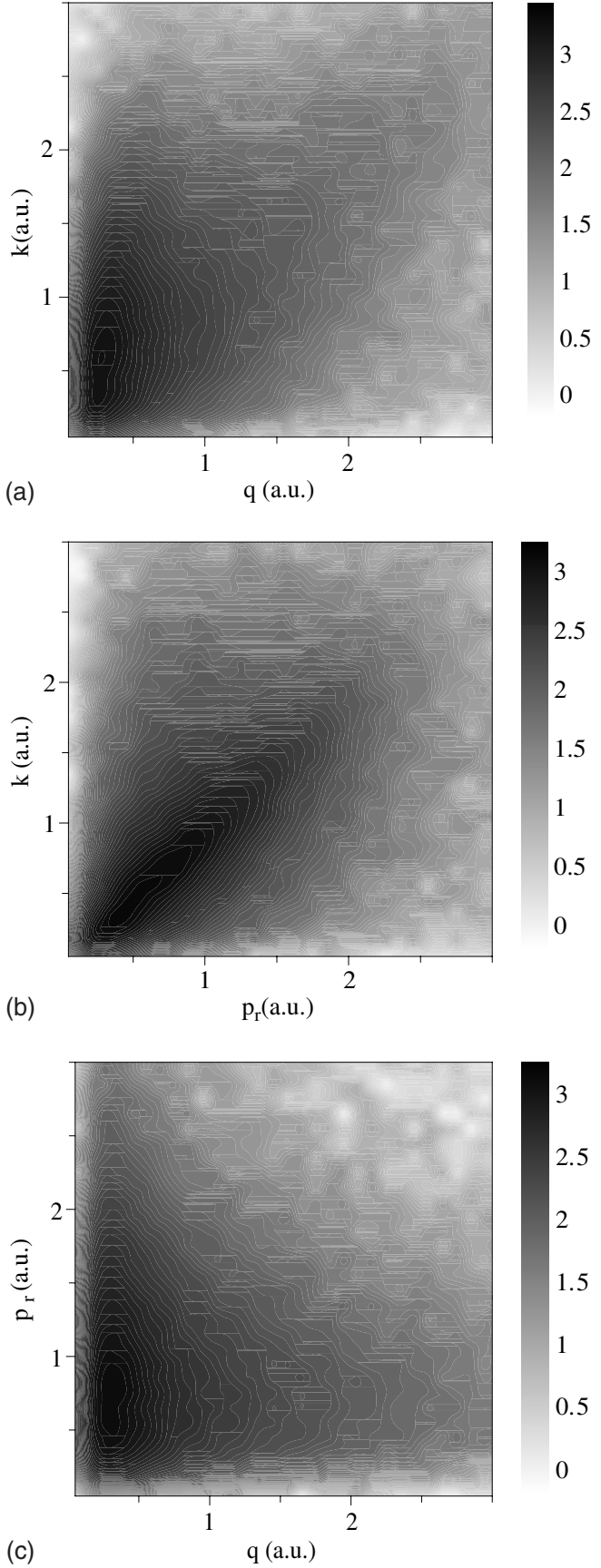

FIG. 2. Experimental doubly differential cross sections (DDCS) for $100 \mathrm{MeV} / \mathrm{amu} \mathrm{C}^{6+}+\mathrm{He}$, extracted from Ref. [1]. (a) DDCS as a function of the magnitudes of the electron momentum $(k)$ and of the projectile momentum transfer $(q)$, (b) DDCS as a function of the magnitudes of the electron $(k)$ and recoil-ion $\left(p_{r}\right)$ momenta, and $(c)$ DDCS as a function of the magnitudes of the recoil-ion momentum $\left(p_{r}\right)$ and of the projectile momentum transfer $(q)$.

From the experimental results that were published in Ref. [1] we have chosen two of the most representative ones to test our theories. Accordingly, in Fig. 4(a) we replicate the experimental $\operatorname{DDCS}(q, k)$ for $3.6 \mathrm{MeV} / \mathrm{amu} \mathrm{Au}^{53+}+\mathrm{He}$. Moreover, the panels (b) and (c) represent the $\operatorname{DDCS}\left(p_{r}, k\right)$ and $\operatorname{DDCS}\left(q, p_{r}\right)$, for the same process, respectively. The theoretical approach is shown in Figs. 5(a)-5(c) using a
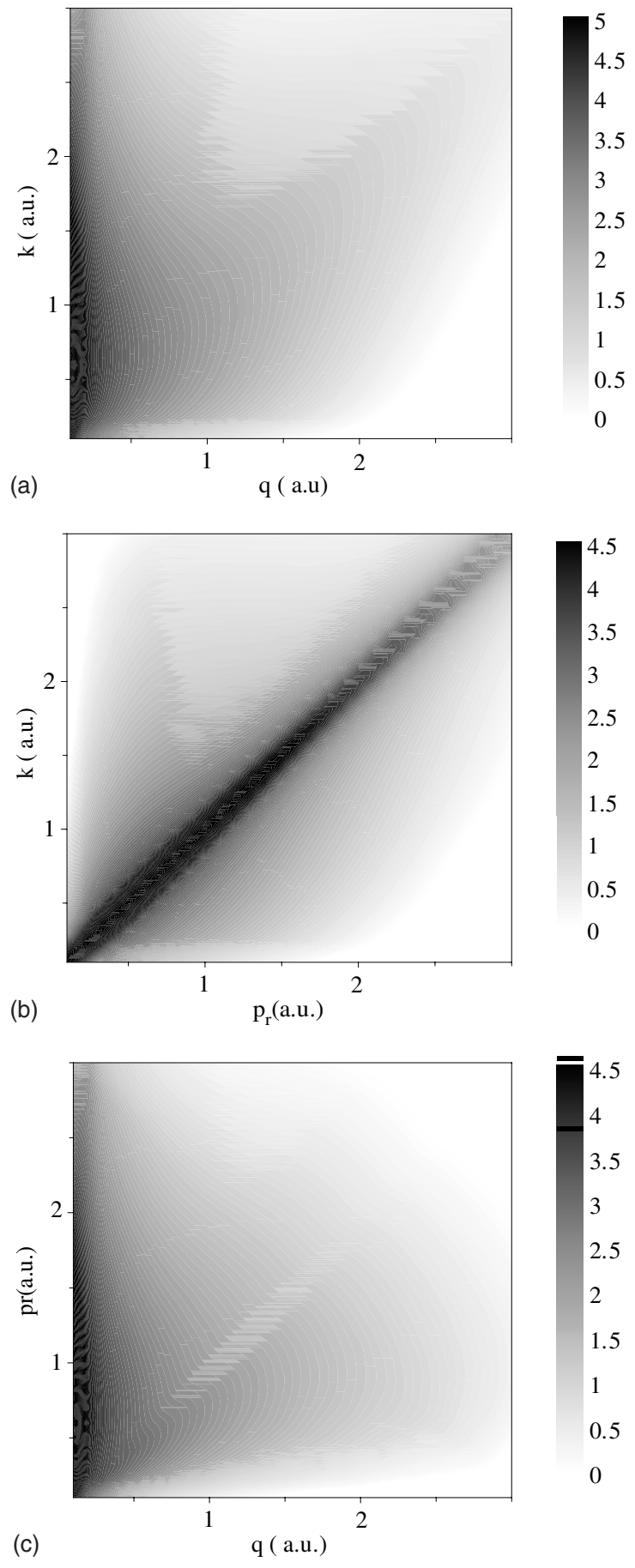

FIG. 3. Theoretical DDCSs for $100 \mathrm{MeV} / \mathrm{amu} \mathrm{C}^{6+}+\mathrm{He}$ using FBA (see the text for details). (a) $\operatorname{DDCS}(q, k)$, (b) $\operatorname{DDCS}\left(p_{r}, k\right)$, and (c) $\operatorname{DDCS}\left(q, p_{r}\right)$.

CDW-EIS theory without taking into account the $N-N$ interaction (see the discussion above).

For the case of $100 \mathrm{MeV} / \mathrm{amu} \mathrm{C}^{6+}+\mathrm{He}$ our theoretical DDCSs in terms of the electron and projectile momenta, i.e., $\operatorname{DDCS}(q, k)$ [Fig. 3(a)], and projectile-recoil-ion variables, i.e., $\operatorname{DDCS}\left(q, p_{r}\right)$ [Fig. 3(c)], respectively, again reproduce the main features found in experimental data [Figs. 2(a) and $2(\mathrm{c})]$. Here, the $\operatorname{DDCS}(q, k)$ is dominated by a stripe-shaped maximum for nearly constant, small $q$, and the $\operatorname{DDCS}\left(q, p_{r}\right)$ 

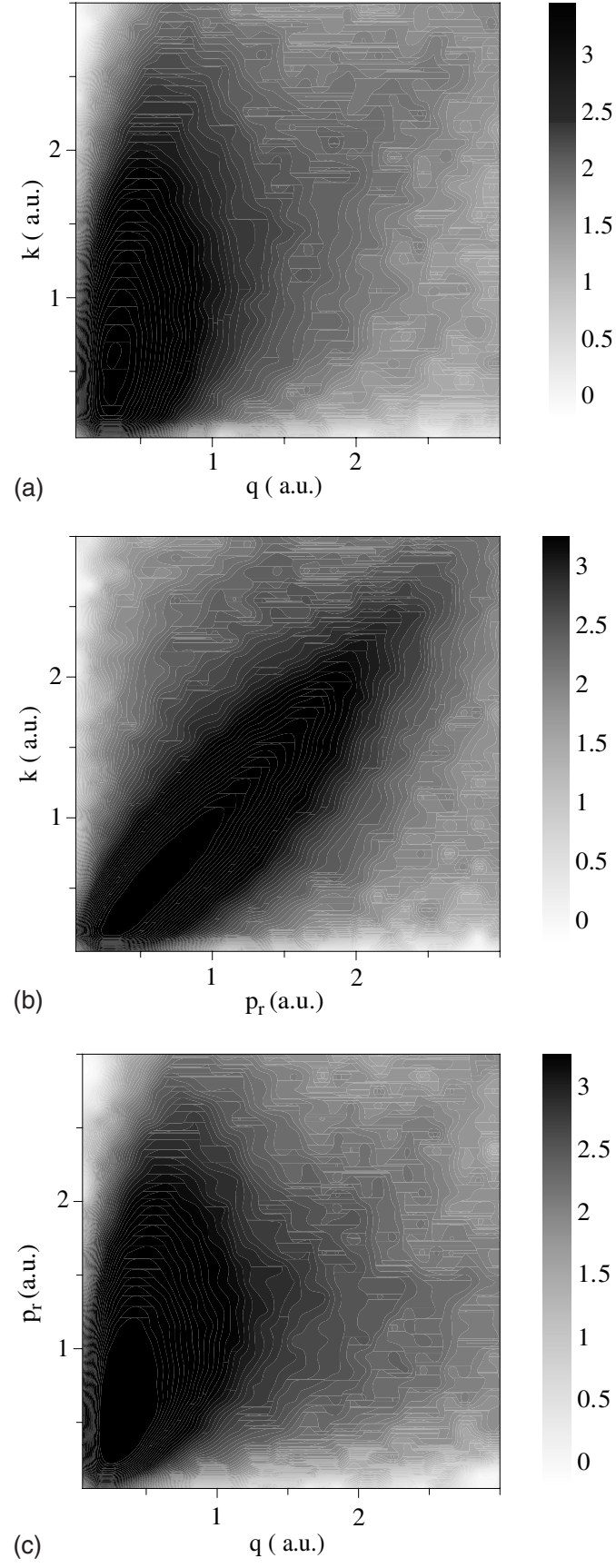

FIG. 4. Idem Fig. 2 for $3.6 \mathrm{MeV} / \mathrm{amu} \mathrm{Au}^{53+}+\mathrm{He}$.

is also governed by a stripe-shaped maximum, which is aligned roughly parallel to the $q$ axis . Additionally, it is possible to see other structures parallel to the $p_{r}$ axis. On the other hand, for $\mathrm{Au}$ projectiles the theoretical approach predicts an enhanced intensity near the diagonal, i.e., strong momentum exchange between ejected electron and projectile and between recoil ion and projectile, Figs. 5(a) and 5(c), respectively, which is not observed in the experimental data [Figs. 4(a) and 4(c)]. Here, this shows that the main underlying ionization mechanisms appear to be different in theory and experiment for the highly charged ion-impact case. Experiments show momentum exchange between the ejected electron and the residual target ion (consistent with a strong field-ionization picture), while theory suggests a stronger ex-
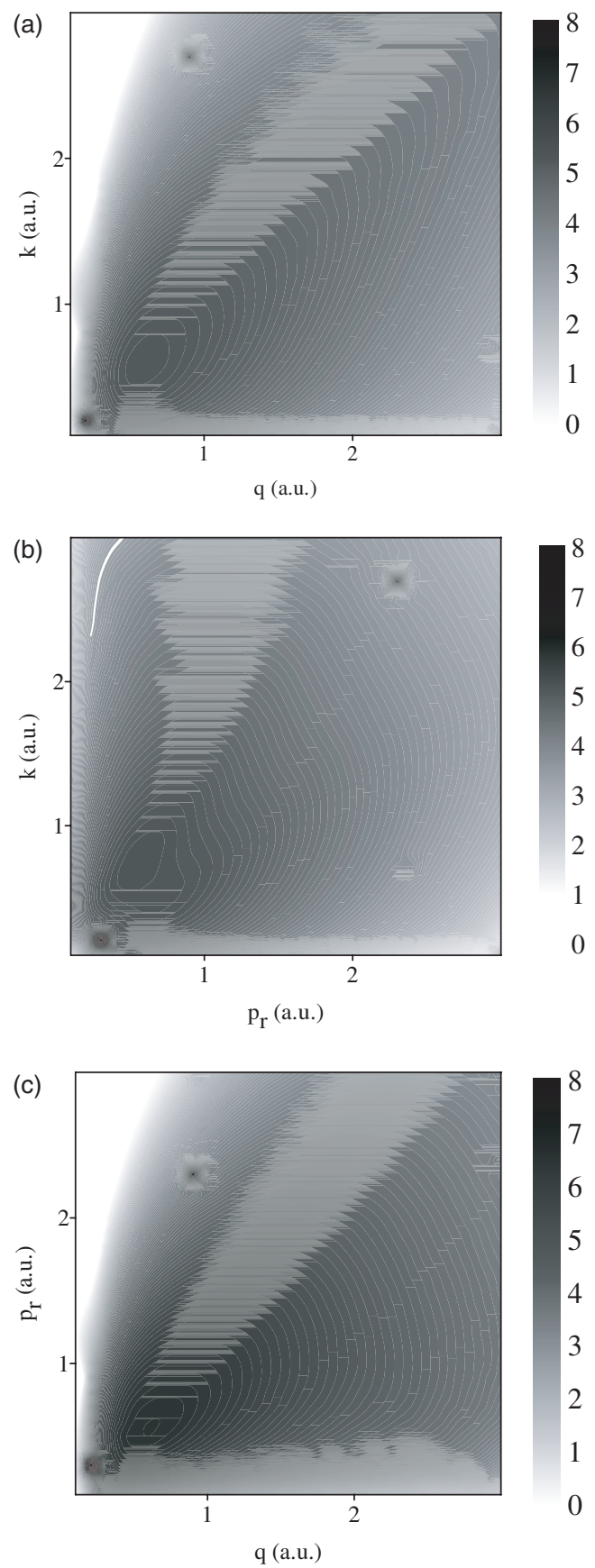

FIG. 5. Theoretical DDCSs for 3.6 MeV/amu $\mathrm{Au}^{53+}+\mathrm{He}$ using CDW-EIS without taking into account $N-N$ interaction (see the text for details). (a) $\operatorname{DDCS}(q, k)$, (b) $\operatorname{DDCS}\left(p_{r}, k\right)$, and (c) $\operatorname{DDCS}\left(q, p_{r}\right)$.

change between the electron and the projectile (a "strong potential Born" picture). The comparison suggests that, for highly charged ion impact, binary-collision mechanisms between the projectile and the electron (or recoil ion), are overestimated by theory. Internuclear interaction is formally taken into account through an eikonal approximation, but it vanishes for these kind of DDCSs. However, little momentum transfer to the electron-recoil-ion subsystem, as observed in the experiments, would require a more symmetric interaction of the projectile with both electron and target nucleus, which is not guaranteed in a standard impact param- 

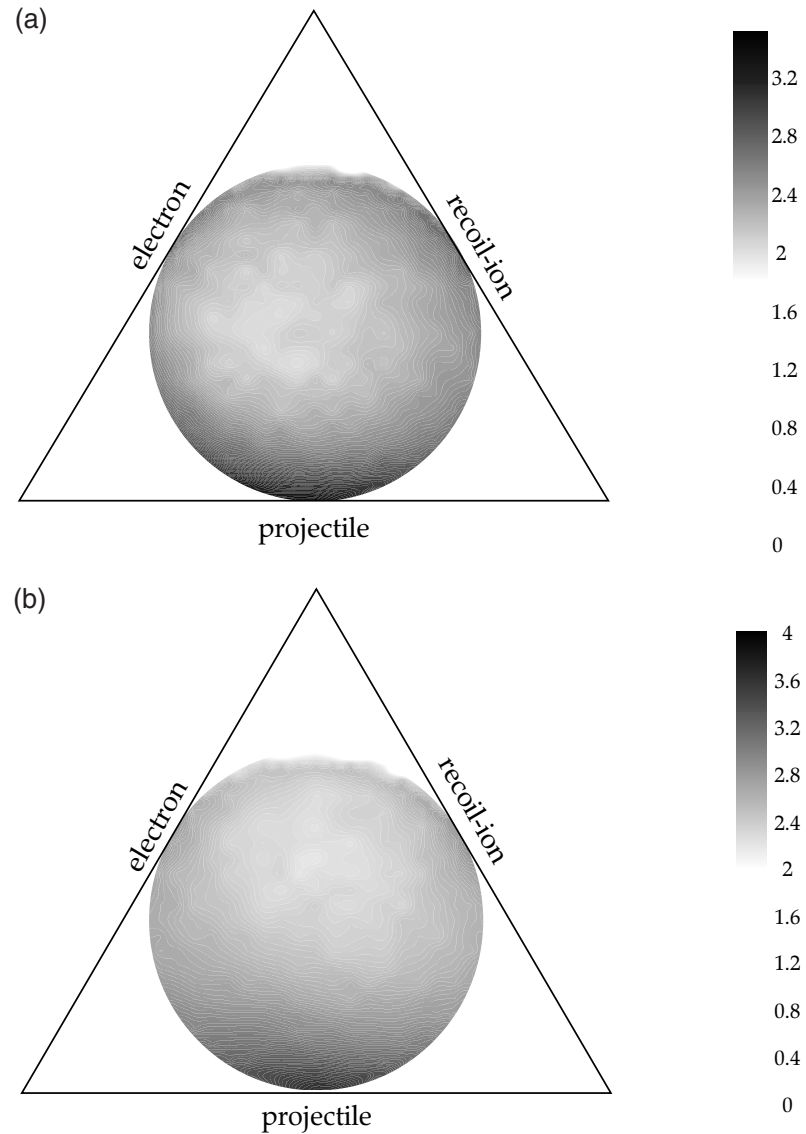

FIG. 6. Experimental Dalitz plots for (a) $100 \mathrm{MeV} / \mathrm{amu} \mathrm{C}^{6+}$ $+\mathrm{He}$ and (b) for $3.6 \mathrm{MeV} / \mathrm{amu} \mathrm{Au}^{53+}+\mathrm{He}$.

eter treatment. These results are consistent with the idea that usual distorted-wave theories such as CDW-EIS fail to accurately describe soft electron emission produced by highly charged ions. The picture that emerges from the experiments is that of a strong-field ionization where there is little net momentum transferred to the whole target atom.

We begin the study of three-particle interactions reproducing in Fig. 6 the experimental Dalitz plot for (a) $100 \mathrm{MeV} / \mathrm{amu} \mathrm{C}^{6+}+\mathrm{He}$ and (b) $3.6 \mathrm{MeV} / \mathrm{amu} \mathrm{Au}^{53+}+\mathrm{He}$. To do a direct comparison with the theories we use the experimental "uncorrelated" graphs (see Ref. [1] for a detailed discussion about correlated and uncorrelated collision events).

In Fig. 7 we show our theoretical results for $100 \mathrm{MeV} / \mathrm{amu} \mathrm{C}^{6+}+\mathrm{He}$ in a Dalitz plot, using (a) first Born approximation and (b) CDW-EIS, without taking into account the internuclear interaction. As discussed in the above paragraphs, the use of a FBA theory is, in principle, justified since for these projectiles the charge-to-velocity ratio is roughly 0.1 (although for certain kinematic conditions significant discrepancies between the FBA and experimental data have been found [5]). We also want to show to what extent a FBA-type theory is able to qualitatively reproduce the features that appear in the experimental data. The large peak at the contact point of the internal circle with the projectile triangle side, which corresponds to $\mathbf{q} \approx \mathbf{0}$, shows again that the strongest two-particle interaction occurs within the
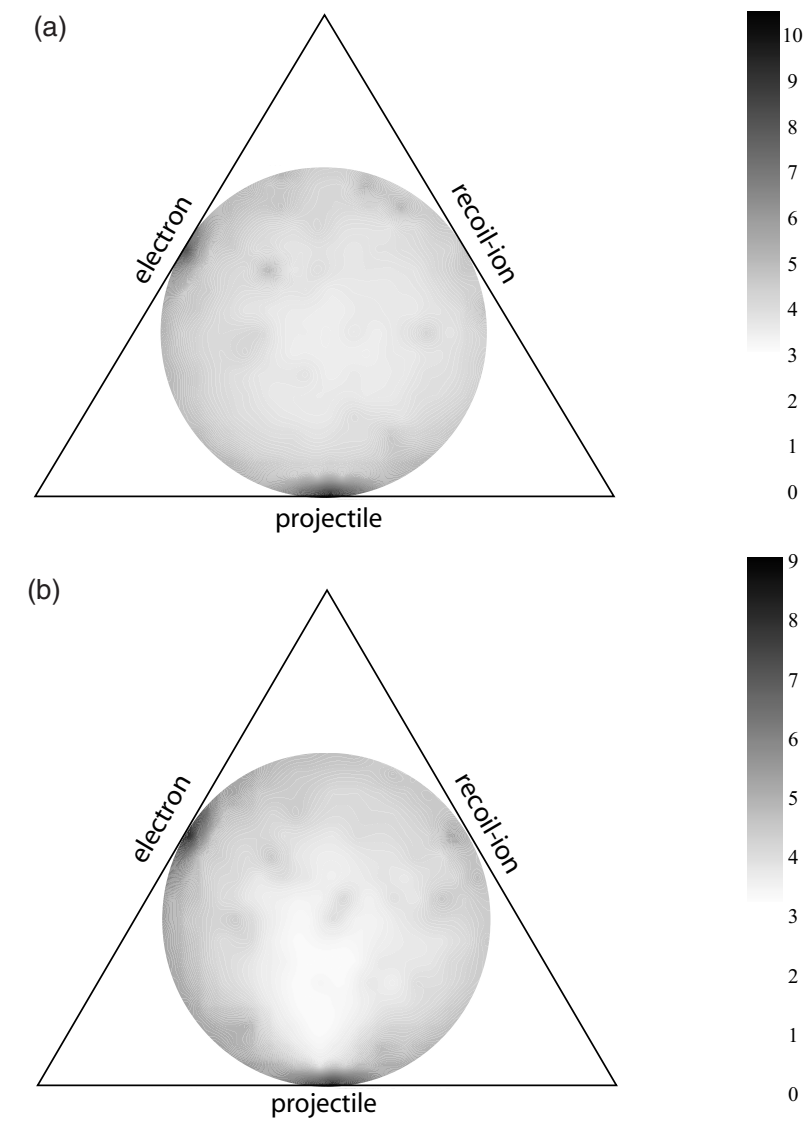

FIG. 7. Theoretical Dalitz plots for $100 \mathrm{MeV} / \mathrm{amu} \mathrm{C}^{6+}+\mathrm{He}$ using (a) FBA and (b) CDW-EIS theory without taking into account the $N-N$ interaction (see text).

subsystem formed by the electron and the recoil ion. Further, a peak at $\pi_{e}=0$ is to be expected since it corresponds to low-energy electron emission. The CDW-EIS results indicate, at $\pi_{e}=0$, a stronger peak than experiments show but, overall, at least for $100 \mathrm{MeV} / \mathrm{amu} \mathrm{C}^{6+}+\mathrm{He}$, both FBA and CDW-EIS theory are able to reproduce qualitatively the main characteristics that the experimental results exhibit.

As Schulz et al. [1] have pointed out, an important feature in the experimental Dalitz plots is a ring close to the rim of the internal circle for the case of $C$ projectiles. For ionization events falling on this rim, two of the three momentum vectors must be pointing opposite to each other while the third momentum vector is either 0 (at the contact points with the triangle sides) or parallel to the momentum vector of the slower of the two former particles. On the other hand, contributions to the inner part of the circle, where all three momenta are similar, were small in the experiment, at least for the uncorrelated data. This shows that three-particle interactions are only significant if they lead to a fragmentation pattern where all three momentum vectors fall on a common line. It should be noted that in the case of the projectiles it is the transferred momentum that is used in the calculation of $\pi_{q}$. This behavior is qualitatively reproduced by our calculations, although the contributions from the inner part of the circle are somewhat larger than in the data. The accumulation of events close to the rim can be understood if the ionization is dominated by electric-dipole transitions. The elec- 

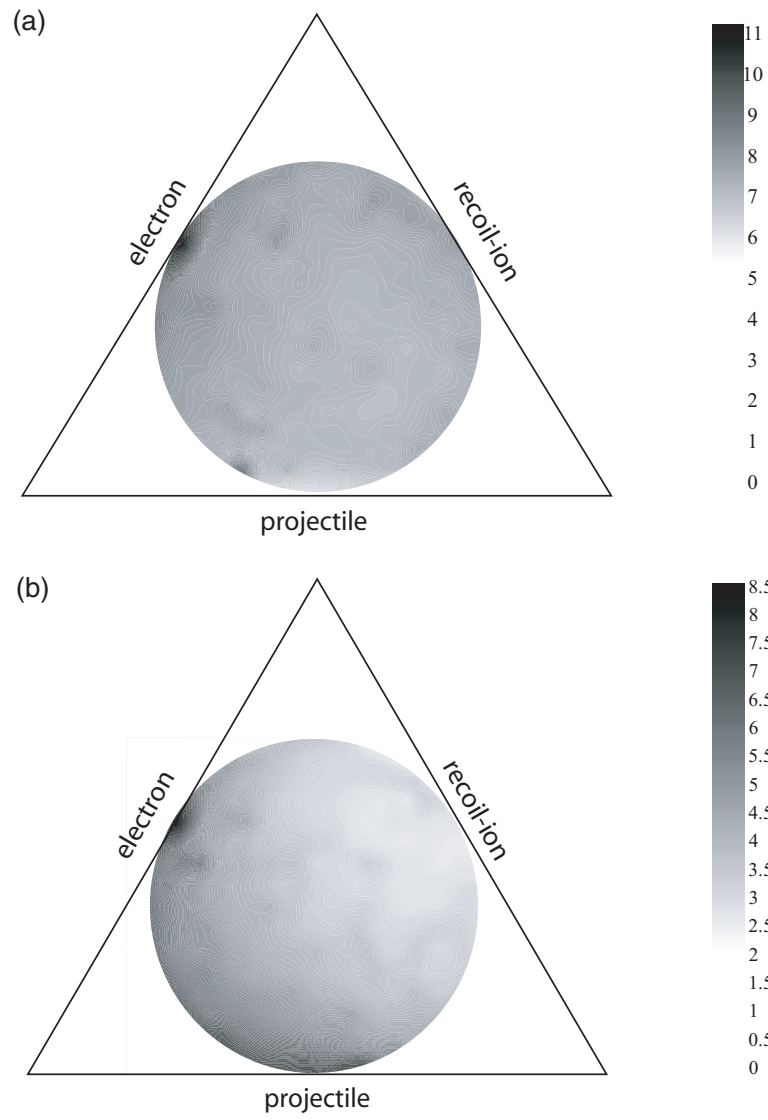

FIG. 8. Theoretical Dalitz plots for $3.6 \mathrm{MeV} / \mathrm{amu} \mathrm{Au}^{53+}+\mathrm{He}$ using (a) CDW-EIS theory without take into account the $N$ - $N$ interaction and (b) CDW-EIS theory including the $N-N$ interaction (see text).

tron angular distribution is then aligned along an axis going through the momentum-transfer vector (i.e., $k$ is parallel or antiparallel to $q$ ). Because of momentum conservation, the recoil-ion angular distribution must then be aligned along the same axis. This behavior is indeed observed in FDCSs (e.g., Ref. [5]).

As low-energy electron emission is dominant, the part of the rim to be populated is that between the $\pi_{e}=0$ and $\pi_{q}$ $=0$ peaks. For a classical head-on binary collision between the electron and the projectile that ends with an electron ejected in the forward direction with $k=2 v_{P}$, we have $k=q$, and the binary peak would correspond to a $p_{r}=0$ peak, which is almost absent here, since we are not looking at highvelocity electrons.

Figure 8 shows theoretical Dalitz plots for $3.6 \mathrm{MeV} / \mathrm{amu}$ $\mathrm{Au}^{53+}+\mathrm{He}$, using a prior CDW-EIS theory without taking into account the $N-N$ interaction (a) and including the $N-N$ interaction in the semiclassical picture as described in Sec. II [panel (b)]. These last results represented a challenge for our numerical calculations, since the theory with $\mathrm{N}-\mathrm{N}$ were made using $10^{6}$ sample points and each plot takes about a week using parallel programming techniques in a cluster formed by several hundred computers. The results show that the theory with the inclusion of $N-N$ interaction qualitatively reproduces the experimental data. More specifically, part of the events near $\pi_{q}=0$, which were removed in the CDW-EIS calculation without the $N-N$ interaction, is restored by the calculation including that interaction. Discrepancies remain; nevertheless, the peak in $\pi_{k}=0$ is observed for the two collision systems studied. However, experiments for $3.6 \mathrm{MeV} / \mathrm{amu} \mathrm{Au}^{24+}+\mathrm{He}$ and $3.6 \mathrm{MeV} / \mathrm{amu} \mathrm{Au}^{53+}+\mathrm{He}$ show a much weaker peak, indicating that the primary mechanism for ionization involves large-momentum exchange between the ejected electron and the residual target, but little momentum exchange with the projectile. We think a better treatment of internuclear interaction, beyond a semiclassical approximation, might be necessary to account for that effect.

Theoretical calculations populate the interior of the circle, which is not observed in the experiments, meaning that large momentum exchange among the three particles appears to be overestimated by theory. This behavior suggests that the description of a three-body wave function in terms of three independent two-body subsystems might suffice for the description of highly charged ion-impact ionization, as Schulz et al. [1] have already suggested.

\section{CONCLUSIONS}

We have presented calculations for ionization DDCSs for highly charged ion impact. We have plotted these DDCSs in terms of ejected-electron momentum, recoil-ion momentum, and projectile-transferred momentum, thus the role of twoparticle interactions is emphasized. In particular, it is shown that for high-projectile charge, an ionization mechanism in which momentum is exchanged mainly between the ejected electron and the recoil ion is underestimated by the standard CDW-EIS theory, probably due to a poor description of the internuclear interaction.

To assess the role of three-particle interactions we calculated theoretical Dalitz plots for $100 \mathrm{MeV} / \mathrm{amu} \mathrm{C}^{6+}+\mathrm{He}$ and 3.6 MeV/amu $\mathrm{Au}^{53+}+\mathrm{He}$, following the experimental data available. We observe that CDW-EIS is able to reproduce the global features of experimental data plotted in the same way. The enhanced importance of the binary projectile-electron and projectile-recoil-ion interactions provides further justification for representing the three-body final state as three independent two-body subsystems, as usually done in distorted-wave theories. In particular, we see that twoparticle interactions seem to dominate the ionization process, suggesting that such a representation of the three-body final state as three independent two-body subsystems may be adequate even for highly charged projectiles. Even with its simplicity, this type of formulation allows for events in which there is significant momentum exchange among the three particles.

\section{ACKNOWLEDGMENTS}

This work has been partially supported by Universidad Nacional del Sur under PGI 24/F038 and by the Agencia Nacional de Promoción Científica y Tecnológica de la República Argentina under PICTO-UNS 931.

\section{APPENDIX}

The aim of this section is to show the variable transformations between $\left(q_{\perp}, \phi_{q}\right)$ and $\left(\pi_{e}, \pi_{q}\right)$ and to emphasize 
their nonlinear nature. We begin rewriting Eqs. (18) and (19),

$$
\begin{aligned}
& \pi_{e}=\frac{k^{2}}{2\left(k^{2}+q^{2}-\mathbf{k} \cdot \mathbf{q}\right)}, \\
& \pi_{q}=\frac{q^{2}}{2\left(k^{2}+q^{2}-\mathbf{k} \cdot \mathbf{q}\right)},
\end{aligned}
$$

and from these two equations directly we can accomplish $q_{\perp}$ as a function of $\left(\pi_{e}, \pi_{q}\right)$, i.e.,

$$
q_{\perp}=\sqrt{\frac{\pi_{q}}{\pi_{e}} k^{2}-q_{z}^{2}} .
$$

Note that $q_{z}$ is a function of $k$, since $q_{z}=\left(k^{2} / 2+\left|\epsilon_{i}\right|\right) / v$.
To obtain $\phi_{q}$ as a function of $\left(\pi_{e}, \pi_{q}\right)$ we begin rearranging Eq. (24) as

$$
\mathbf{k} \cdot \mathbf{q}=\frac{k^{2}}{\pi_{e}}\left(\pi_{e}+\pi_{q}-\frac{1}{2}\right)
$$

and defining $k_{\perp}=k \sin \theta_{k}$ and $k_{z}=k \cos \theta_{k}$ Eq. (27) yields

$$
q_{\perp} k_{\perp} \cos \left(\phi_{k}-\phi_{q}\right)=\frac{k^{2}}{\pi_{e}}\left(\pi_{e}+\pi_{q}-\frac{1}{2}\right)-q_{z} k_{z} .
$$

Finally, we obtain

$$
\cos \left(\phi_{k}-\phi_{q}\right)=q_{\perp} k_{\perp}^{-1}\left[\frac{k^{2}}{\pi_{e}}\left(\pi_{e}+\pi_{q}-\frac{1}{2}\right)-q_{z} k_{z}\right] .
$$

From this last equation we can obtain $\phi_{q}$ as a function of the other variables.
[1] M. Schulz, R. Moshammer, D. Fischer, and J. Ullrich, J. Phys. B 37, 4055 (2004).

[2] R. Moshammer, J. Ullrich, M. Unverzagt, W. Schmitt, P. Jardin, R. E. Olson, R. Mann, R. Dörner, V. Mergel, and U. Buck et al., Phys. Rev. Lett. 73, 3371 (1994).

[3] M. Schulz, R. Moshammer, D. H. Madison, R. E. Olson, P. Marchalant, C. T. Whelan, H. R. J. Walters, S. Jones, M. Foster, H. Kollmus et al., J. Phys. B 34, L305 (2001).

[4] D. H. Madison, D. Fischer, M. Foster, M. Schulz, R. Moshammer, S. Jones, and J. Ullrich, Phys. Rev. Lett. 91, 253201 (2003).

[5] M. Schulz, R. Moshammer, D. Fischer, H. Kollmus, D. Madison, S. Jones, and J. Ullrich, Nature (London) 422, 48 (2003).

[6] D. R. Bates and G. W. Griffing, Proc. Phys. Soc., London, Sect. A 66, 961 (1953).

[7] H. Ehrhardt, K. Jung, G. Knoth, and P. Schlemmer, Z. Phys. D: At., Mol. Clusters 1, 3 (1986), and references therein.

[8] F. W. Byron, C. J. Joachain, and B. Piraux, J. Phys. B 13, L673 (1980).

[9] P. Marchalant, C. T. Whelan, and H. R. J. Walters, J. Phys. B 31, 1141 (1998).

[10] Z. J. Chen, D. H. Madison, C. T. Whelan, and H. R. J. Walters, J. Phys. B 37, 981 (2004).

[11] D. Belkić, J. Phys. B 11, 3529 (1978).

[12] I. M. Cheshire, Proc. Phys. Soc. Jpn. 84, 89 (1964).

[13] D. S. F. Crothers, J. Phys. B 15, 2061 (1982).

[14] D. S. F. Crothers and J. F. McCann, J. Phys. B 16, 3229 (1983).

[15] P. D. Fainstein, V. H. Ponce, and R. D. Rivarola, J. Phys. B 24, 3091 (1991).

[16] N. Stolterfoht, R. D. Dubois, and R. D. Rivarola, Electron Emission in Heavy Ion-Atom Collisions (Springer, Berlin, 1997).

[17] L. Gulyás, P. D. Fainstein, and A. Salin, J. Phys. B 28, 245 (1995).
[18] L. Gulyás and P. D. Fainstein, J. Phys. B 31, 3297 (1998).

[19] V. D. Rodríguez and R. O. Barrachina, Phys. Rev. A 57, 215 (1998).

[20] M. D. Sánchez, W. R. Cravero, and C. R. Garibotti, Phys. Rev. A 61, 062709 (2000).

[21] R. McCarroll and A. Salin, J. Phys. B 11, L693 (1978).

[22] M. Foster, D. H. Madison, J. L. Peacher, M. Schulz, S. Jones, D. F. D. R. Moshammer, and J. Ullrich, J. Phys. B 37, 1565 (2004).

[23] R. T. Pedlow, S. F. C. O'Rourke, and D. S. F. Crothers, Phys. Rev. A 72, 062719 (2005).

[24] I. Bray and A. T. Stelbovics, Phys. Rev. Lett. 69, 53 (1992).

[25] R. E. Olson and A. Salop, Phys. Rev. A 16, 531 (1977).

[26] J. Ullrich, R. Moshammer, A. Dorn, L. Schmidt, and H. Schmidt-Böcking, Rep. Prog. Phys. 66, 1463 (2003).

[27] M. Schulz, R. Moshammer, W. Schmitt, H. Kollmus, R. Mann, S. Hagmann, R. E. Olson, and J. Ullrich, Phys. Rev. A 61, 022703 (2000).

[28] S. Otranto, R. E. Olson, and J. Fiol, J. Phys. B 39, L175 (2006).

[29] M. F. Ciappina, W. R. Cravero, and C. R. Garibotti, J. Phys. B 36, 3775 (2003).

[30] H. Bethe, Ann. Phys. 5, 325 (1930).

[31] M. Inokuti, Rev. Mod. Phys. 43, 297 (1971).

[32] J. Berakdar, J. S. Briggs, and H. Klar, J. Phys. B 26, 285 (1993).

[33] E. Clemente and C. Roetti, At. Data Nucl. Data Tables 14, 177 (1974).

[34] L. Rosenberg, Phys. Rev. D 8, 1833 (1973).

[35] C. R. Garibotti and J. E. Miraglia, Phys. Rev. A 21, 572 (1980).

[36] D. S. F. Crothers and L. J. Dubé, Adv. At., Mol., Opt. Phys. 30, 287 (1992).

[37] R. H. Dalitz, Philos. Mag. 44, 1068 (1953). 